\title{
Trait approach motivation moderates the aftereffects of self-control
}

\section{Adrienne Crowell, Nicholas J. Kelley and Brandon J. Schmeichel*}

Department of Psychology, Texas A\&M University, College Station, TX, USA

\section{Edited by:}

Jacob B. Hirsh, University of Toronto Canada

\section{Reviewed by:}

Virgil Zeigler-Hill, Oakland University, USA

Dylan D. Wagner, Ohio State

University, USA

\section{${ }^{*}$ Correspondence:}

Brandon J. Schmeichel, Department of Psychology, Texas A\&M University, College Station, TX 77843-4235, USA e-mail: schmeiche/@tamu.edu
Numerous experiments have found that exercising self-control reduces success on subsequent, seemingly unrelated self-control tasks. Such evidence lends support to a strength model that posits a limited and depletable resource underlying all manner of self-control. Recent theory and evidence suggest that exercising self-control may also increase approach-motivated impulse strength. The two studies reported here tested two implications of this increased approach motivation hypothesis. First, aftereffects of self-control should be evident even in responses that require little or no self-control. Second, participants higher in trait approach motivation should be particularly susceptible to such aftereffects. In support, exercising self-control led to increased optimism (Study 1) and broadened attention (Study 2), but only among individuals higher in trait approach motivation. These findings suggest that approach motivation is an important key to understanding the aftereffects of exercising self-control.

Keywords: approach motivation, ego depletion, self-control, self-regulation, individual differences

\section{TRAIT APPROACH MOTIVATION MODERATES THE AFTEREFFECTS OF SELF-CONTROL}

Exercising self-control can be a frustrating chore. The classic illustration of self-control involves resisting the temptation of an immediate pleasure (e.g., eating a donut) in the hopes of gaining some future benefit (e.g., well-fitting clothes). More generally, self-control refers to purposeful efforts to override or alter a predominant response tendency. People routinely apply self-control to conquer diverse challenges in life including losing weight, breaking habits, managing emotions, and persisting at difficult tasks (e.g., Hofmann et al., 2012b).

According to the strength model of self-control (Muraven and Baumeister, 2000), overriding a response consumes and depletes a limited inner resource, thereby causing a short-term deficit in self-control. Experiments inspired by the strength model have used a sequential task paradigm in which participants perform two self-control challenges in succession. The guiding question has been: does performing one self-control task undermine performance on a subsequent self-control task? The answer has been a convincing yes. Over 100 experiments have found that initial acts of self-control undermine performance on subsequent, seemingly unrelated self-control challenges (i.e., the ego depletion effect; for a meta-analysis, see Hagger et al., 2010). For example, resisting tempting chocolate treats can reduce persistence at a difficult puzzle (Baumeister et al., 1998), and focusing attention narrowly for an extended period of time can increase aggressive responding to an insult (Finkel et al., 2009). This evidence strongly suggests that prior exertions of self-control temporarily increase the likelihood of self-control failure.

The current investigation asked a different question about the consequences of exercising self-control. Rather than assessing performance on the second of two self-control tasks, we assessed responses on a second task that required little or no self-control. Based on a process model proposing that exercising self-control causes temporary shifts in motivation and attention (Inzlicht and Schmeichel, 2012; Inzlicht et al., 2014), we predicted that initial acts of self-control may influence subsequent responses that reflect approach motivation, even if such responses do not require self-control.

\section{SELF-CONTROL AND APPROACH MOTIVATION}

Self-control can be construed as a competition between two opposing forces: the motivational force of an impulse (i.e., impulse strength) versus the countervailing force that overrides the impulse (i.e., self-control strength). In this view, self-control succeeds when the impulse is relatively weak, when control is relatively strong, or through some combination of both of these factors (e.g., Carver, 2005; Hofmann et al., 2009). Conversely, failures of self-control may stem from strong impulses, weak self-control strength, or a combination of both factors.

Research on the strength model has focused on the control side of this equation, and the prevailing interpretation of the ego depletion effect is that exercising self-control temporarily reduces self-control strength. But several findings in the ego depletion literature may also be explained by increased impulse strength, rather than or in addition to reduced self-control strength. For example, Vohs and Heatherton (2000) observed that suppressing emotional reactions causes dieters to overeat ice cream. This finding suggests that suppressing emotions temporarily reduces the capacity to control the consumption of unhealthy food, consistent with the strength model. But on logical grounds it is also possible that exercising self-control increased dieters' motivation to eat the ice cream. The outcome-more ice cream consumption - by itself is not sufficient to determine whether weak control, strong impulses, or a combination of 
both factors was the cause. This ambiguity also applies to other approach-motivated responses that have been found to increase after exercising self-control. Approach motivation refers to the motivation to go toward a stimulus (Harmon-Jones et al., 2013). Approach motivation is closely associated with reward sensitivity (i.e., more interest in and stronger responding to rewarding stimuli), but it has also been associated with aggression and anger (Carver and Harmon-Jones, 2009). Prior research has observed that exercising self-control increases several approach-motivated responses including aggressive responding to provocation (e.g., Stucke and Baumeister, 2006; DeWall et al., 2007; Denson et al., 2011), confirmatory information processing (Fischer et al., 2008), increased alcohol consumption (Muraven et al., 2002; Christiansen et al., 2012), and increased risk-taking (Freeman and Muraven, 2010).

Based on this evidence and additional findings that are not readily explained by a strength model of self-control, Inzlicht and Schmeichel (2012) proposed a process model of ego depletion. According to this model, exercising selfcontrol is an aversive activity that causes shifts in motivation toward increased reward sensitivity and away from further efforts at self-control. Unlike the strength model, the process model presumes that exercising self-control influences subsequent performance on both self-control tasks and non-controlled responses.

This process model received initial support in research testing the hypothesis that exercising self-control increases approach motivation (Schmeichel et al., 2010). In a first experiment, participants either suppressed or expressed their emotions during a picture-viewing task before completing a self-report measure of approach motivation. Participants who had suppressed their emotions reported higher levels of approach motivation, compared to participants who had not suppressed their emotions. In a second experiment, participants who had (versus had not) inhibited the use of two common letters on a writing task went on to take more risks on a low-stakes gambling game.

The results from Schmeichel et al. (2010) were consistent with the prediction that exercising self-control increases approach motivation, and they provided initial evidence that the aftereffects of self-control can extend to responses that are not targeted for control. However, one could question whether the dependent measures they used were sensitive mainly to variations in approach motivation or whether other variables (e.g., selfcontrol) may also have played a role, and their findings did not address mechanisms underlying the observed effects. The current investigation sought evidence that individual differences in approach motivation moderate the aftereffects of self-control, consistent with the assumption that reward sensitivity is an important key to understanding the consequence of exercising self-control.

\section{TRAIT APPROACH MOTIVATION AS A MODERATOR}

One accepted method for elucidating the process underlying an experimental effect is to examine the role of individual difference variables that influence the tendency to engage the proposed process (e.g., Gohm and Clore, 2000; see also Underwood,
1975). Examining whether such individual differences moderate an experimental effect is a way to test assumptions about the processes or mechanism underlying the effect.

At issue in the current investigation is the effect of exercising self-control on non-controlled responses, and the process presumed to underlie this effect is a shift in motivational orientation toward increased reward sensitivity. Although approach motivation encompasses the tendency to move toward all types of positive stimuli, individual differences in approach motivation have been linked explicitly to variations in reward sensitivity (e.g., Carver and White, 1994; Beaver et al., 2006). Evidence that trait approach motivation moderates the effects of exercising self-control would thus lend support to the idea that a shift in motivational orientation toward increased reward sensitivity is one process by which exercising self-control influences subsequent responding.

Because we were interested in the aftereffects of self-control for responses that themselves require very little self-control, we did not anticipate main effects of our experimental manipulations. Although many prior experiments on ego depletion have found main effects of exercising versus not exercising self-control (Hagger et al., 2010), those studies have used self-control tasks as dependent measures (and even some of these have found that ego depletion is moderated by individual differences; e.g., Gröpel et al., 2014; Salmon et al., 2014). The few studies that have assessed the effects of ego depletion on the performance of non-self-control tasks (as do the present studies) have observe null effects (e.g., Schmeichel et al., 2003; Schmeichel, 2007; cf. Schmeichel et al., 2010). Thus, main effects of the ego depletion manipulations seemed unlikely in the current studies. We adopted the more circumscribed prediction that the aftereffects of exercising selfcontrol would emerge mainly among participants prone to higher reward sensitivity (i.e., those higher in trait approach motivation). Evidence of moderation by trait approach motivation would suggest that one process underlying the aftereffects of self-control (at least for the non-self-control tasks used in the current experiments) is increased reward sensitivity. To increase confidence in this conclusion, we also tested for moderation by trait self-control. If changes in self-control are crucial for any observed effects (as would be assumed by the strength model), then any aftereffects of exercising self-control should be moderated by trait self-control and not by trait approach motivation.

\section{THE CURRENT STUDIES}

Two studies tested the hypothesis that exercising self-control influences approach-related responding, particularly among individuals higher in trait approach motivation. Each study included an established measure of individual differences in approach motivation, namely the behavioral inhibition system and behavioral activation system scales (BIS/BAS; Carver and White, 1994), and an established measure of trait self-control (i.e., the trait self-control scale; Tangney et al., 2004). Each study also included a writing task to manipulate the exercise of self-control (borrowed from Schmeichel, 2007; see also Mead et al., 2009; Schmeichel and Vohs, 2009; Lewandowski et al., 2012).

The main difference across studies was the dependent measure. Each study included a dependent measure that has been associated with approach-related states in previous research, but 
has not previously been associated with states associated with self-control. Specifically, Study 1 examined optimism because prior research found that the approach-related feelings of anger (e.g., Lerner et al., 2003) and power (e.g., Anderson and Galinsky, 2006) are associated with increased optimism. Study 2 examined attentional breadth because previous research found that experimentally induced increases in approach motivation (Förster et al., 2006) and in positive mood states associated with approach motivation (Gasper and Clore, 2002) cause an increase in attentional breadth. Note that we do not consider measures of optimism or of attentional breadth to be direct measures of approach motivation. Rather, they are measures that have proven sensitive to experimental manipulations of approach-motivated states in previous research. Accordingly, we included them as dependent measures in the current studies to test the hypothesis that exercising self-control temporarily increases reward sensitivity, and especially so among individuals higher in trait approach motivation.

We predicted that exercising self-control on the writing task, much like the manipulations of approach-related states mentioned above, would induce an approach-oriented state particularly among individuals higher in trait approach motivation, and as a result increase optimism and attentional breadth, respectively. Evidence to support the hypothesized interaction between exercising self-control and trait approach motivation would suggest that approach motivation helps to shape the aftereffects of self-control. Furthermore, insofar as the aftereffects of exercising self-control are moderated by trait approach motivation but not trait self-control, this would clearly suggest that approach motivation-not selfcontrol-is crucial for understanding some aftereffects of selfcontrol.

\section{STUDY 1}

Study 1 tested the hypothesis that exercising self-control causes a subsequent increase in optimism that suggests heightened approach motivation (e.g., Lerner etal., 2003; Anderson and Galinsky, 2006). Participants completed a personality test and wrote a story that did versus did not require them to inhibit the use of specific letters. Then participants indicated the likelihood of experiencing several negative health-related outcomes. Last, they completed a questionnaire measure of trait approach motivation. We predicted an interaction between approach motivation and the writing manipulation, such that participants higher in trait approach motivation exhibit more optimism after exerting self-control on the writing task.

\section{METHOD}

\section{Ethics statement}

The study was approved by the Institutional Review Board (IRB) at Texas A\&M University. A waiver of informed consent was approved by the IRB because the study involved no more than minimal risk to participants. Verbal informed consent was obtained from all participants after they read an information sheet (essentially an informed consent form that does not ask for a signature). If they consent, they were allowed to participate. Therefore, their data reflects documentation of their consent.

\section{Participants and design}

Thirty-nine undergraduate students (27 women, and 12 men; age $M=18.23, \mathrm{SD}=0.48$ ) reported individually to a laboratory study described as an investigation of the relationships among personality traits and cognitive abilities. They received credit toward a course requirement for their participation. One additional participant completed the study but was not included in analyses because she circled all zeroes on the dependent measure. Participants were randomly assigned between the free writing $(n=19)$ and controlled writing $(n=20)$ conditions.

\section{Procedure}

The experimenter introduced the purpose of the study before having participants (in keeping with the cover story) complete a bogus personality assessment (borrowed from Sedikides and Green, 2000). Then participants spent $5 \mathrm{~min}$ writing a short autobiographical story. To manipulate the exercise of self-control, participants were randomly assigned to write the story in one of two ways (Schmeichel, 2007). Participants in the free writing condition were instructed simply to "Write a story about a recent trip you have taken. It may be a trip to the store, to Ohio, or to another country - wherever! Just pick something that is relatively fresh in your memory." Participants in the controlled writing condition received an additional instruction: "Very important! Do not use the letters A or $\mathrm{N}$ anywhere in your story! If you find yourself writing a word that includes the letters $\mathrm{A}$ or $\mathrm{N}$, please stop writing that word and find an alternate way to express your thoughts." Hence, one group was required to control their writing by avoiding the use of two common letters, whereas the other group wrote without restrictions.

After the writing manipulation, all participants judged the personal likelihood of six different negative health outcomes borrowed from Perloff and Fetzer (1986; e.g., "You will be diagnosed with cancer"; "You will develop diabetes;" $\alpha=0.71)$ using a scale from 0 (very unlikely) to 10 (very likely). The mean response to the six health outcome items (reverse-scored so that higher scores indicate more optimism) served as our primary dependent measure (sample $M=7.85, \mathrm{SD}=1.50, \alpha=0.71$ ).

Then participants completed a well-validated measure of behavioral inhibition and behavioral activation system sensitivities (the BIS/BAS scales; Carver and White, 1994) Participants responded to BIS/BAS items using a scale from 1 (very false for $m e$ ) to 4 (Very true for me). The BAS scale included 13 items assessing desire for reward, positive responses to real or anticipated reward, and persistence in pursuing desired reward. Sample items include "I go out of my way to get things I want," and "I often act on the spur of the moment." In the current study the average total score on the BAS scale was $M=40.72$ ( $\mathrm{SD}=4.95, \alpha=0.77)$. The seven-item BIS scale assessed threat sensitivity ("Criticism or scolding hurts me quite a bit") and was included to explore possible contributions of trait BIS sensitivity to the effects of exercising self-control on health optimism. The average score on the BIS scale was $M=21.77$ ( $\mathrm{SD}=4.13$, $\alpha=0.83$ ).

Last, participants completed the brief version of the trait selfcontrol scale (Tangney et al., 2004). This scale included 13 items assessing ability to override impulses and exercise self-control and 
was included to verify that individual differences in approach motivation, not self-control ability, moderate changes in optimism under ego depletion. Sample items include "I refuse things that are bad for me," and "I am good at resisting temptation." Participants responded to each item using a scale from 1 (not at like me) to 5 (very much like me). The average score on the trait self-control scale in the current sample was $M=42.92(\mathrm{SD}=8.83, \alpha=0.84)^{1}$. Correlations among variables in this study are displayed in Table 1.

\section{RESULTS}

The central hypothesis was that the controlled writing task would increase optimism particularly among participants higher (versus lower) in trait approach motivation. It did. We regressed optimism scores (i.e., the average response to the six negative health-related items) on writing condition, BAS scores (centered), and the Writing $\times$ BAS interaction. Neither the main effect of BAS, $b=-0.03$, $t(37)=0.75, p=0.46$, nor the main effect of writing condition, $b=0.71, t(37)=1.73, p=0.09$, was statistically significant. More importantly, the predicted Writing $\times$ BAS interaction was statistically significant and in line with predictions, $b=0.22, t(37)=2.72$, $p=0.01$.

Simple slopes analyses indicated that among participants higher in BAS, the controlled writing condition caused an increase in optimism relative to the free writing condition, $b=1.77$, $t(37)=3.34, p=0.003$. Among participants lower in BAS, the writing manipulation did not influence optimism, $t(37)<1$. Figure 1 displays the optimism scores based on predicted values at higher (1 SD above the mean) versus lower levels (1 SD below the mean) of BAS.

We ran the regression again, this time with trait self-control as the individual difference predictor instead of BAS. More specifically, we regressed optimism scores on writing condition, trait self-control (centered), and the Writing $\times$ Trait Self-Control interaction. The main effect of writing condition was not statistically significant, $b=0.35, t(37)=0.89, p=0.38$, but the main effect of trait self-control was statistically significant, $b=0.06, t(37)=2.81$, $p=0.01$, such that participants reporting higher trait self-control

${ }^{1}$ Because the BIS/BAS and trait self-control measures were completed at the end of the study, we checked to see if scores differed across experimental conditions. We found no differences in BIS/BAS, as a function of condition, $t \mathrm{~s}(37)<1$. However, participants in the controlled writing condition $(M=45.65, \mathrm{SD}=6.48)$ unexpectedly reported more trait self-control than did participants in the free writing condition $(M=40.05, \mathrm{SD}=10.16), t(37)=2.06, p=0.05$.

Table 1 | Correlations among the individual difference variables and optimism in Study 1.

\begin{tabular}{lllll}
\hline Variable & $\mathbf{1}$ & $\mathbf{2}$ & $\mathbf{3}$ & $\mathbf{4}$ \\
\hline 1. BAS & - & -0.08 & $-0.36^{*}$ & -0.12 \\
2. BIS & & - & 0.21 & 0.13 \\
3. Trait self-control & & & - & $0.47^{* *}$ \\
4. Optimism & & & & - \\
\hline
\end{tabular}

Optimism $=$ the average response to the six negative health-related items. ${ }^{*} p<0.05,{ }^{*} p<0.001$.

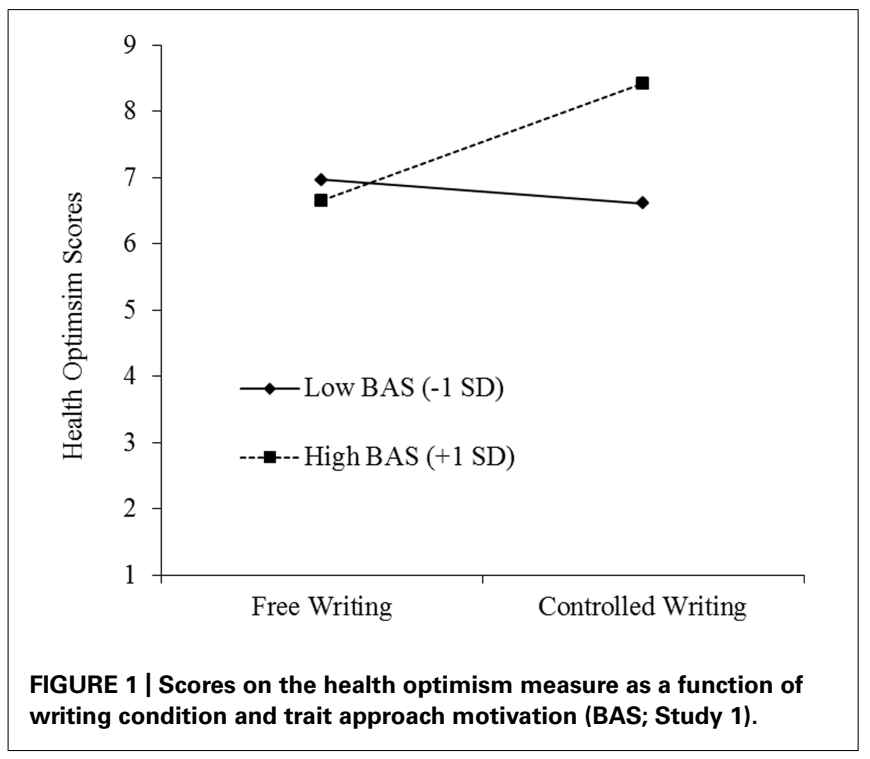

also expressed greater optimism. More importantly, the Writing $\times$ Trait Self-Control interaction fell short of conventional levels of statistical significance, $b=-0.09, t(37)=1.76, p=0.09$.

With BIS as the individual difference predictor, neither the main effect of writing condition, $b=0.67, t(37)=1.59, p=0.12$, nor the main effect of BIS, $b=0.03, t(37)=0.48, p=0.63$, was statistically significant. Further, neither was the Writing $\times$ BIS interaction, $b=-0.16, t(37)=1.53, p=0.14$.

\section{DISCUSSION}

The results from Study 1 revealed that exercising self-control makes some individuals more optimistic about future healthrelated outcomes. Specifically, individuals higher in trait approach motivation judged various negative health outcomes as less likely to happen to them after exercising (versus not exercising) selfcontrol on a writing task. Individuals lower in trait approach motivation showed no such effect of exercising self-control. These results support the hypothesis that exercising self-control increases optimistic health perceptions in a manner suggesting increased approach motivation.

The aftereffects of exercising self-control on optimism were not moderated by trait self-control, which suggests that selfcontrol did not underlie the observed effects. However, at least two patterns of findings caution against discounting the role of self-control in optimism. First, the zero-order correlation between trait self-control and optimism was statistically significant, such that persons higher in trait self-control reported more healthrelated optimism. Second, although the interaction between the trait self-control and the writing manipulation was not statistically significant, it was in the range that some investigators may consider marginally significant. Together these unexpected results hint that both BAS and trait self-control may be relevant to the effects of ego depletion on optimism. Thus, although trait BAS but not trait self-control interacted with the writing manipulation to influence optimism as we had predicted, it seems premature to conclude that trait self-control was irrelevant to the observed results. 
Three additional limitations of the study should be noted. First, Study 1 examined only optimistic health perceptions as the dependent measure. Optimism has been associated with approach-related states in previous research, but if our hypothesis is correct, then people higher in trait approach motivation should exhibit increases in other types of approach-related responses after exercising self-control. Second, Study 1 was underpowered due to its small sample size. Third, in Study 1 the measures of trait selfcontrol, BAS, and BIS were administered at the end of the study. This timing of events is suboptimal insofar as trait self-control scores varied as a function of experimental condition, which suggests that the writing manipulation may have influenced reports of trait self-control. (The direction of influence was in the opposite direction of the ego depletion effect such that participants reported higher trait self-control following the controlled writing task). Therefore, Study 2 included the trait measures prior to the experimental manipulation, tested a larger sample of participants, and featured a different dependent measure.

\section{STUDY 2}

Study 2 tested the hypothesis that exercising self-control causes a subsequent increase in attentional breadth in a manner that reveals heightened approach motivation (Gasper and Clore, 2002; Förster et al., 2006; cf. Gable and Harmon-Jones, 2008). Participants first completed questionnaire measures of BAS, BIS, and trait selfcontrol and then wrote a story that did versus did not require them to inhibit the use of two commonly used letters. Then participants performed a local/global processing task. We predicted an interaction between approach motivation and the writing manipulation, such that participants higher in trait approach motivation show a more pronounced global processing bias following the controlled writing task.

\section{METHOD}

\section{Ethics statement}

The study was approved by the IRB at Texas A\&M University. A waiver of informed consent was approved by the IRB because the study involved no more than minimal risk to participants. Verbal informed consent was obtained from all participants after they read an information sheet (essentially an informed consent form that does not ask for a signature). If they consent, they were allowed to participate. Therefore, their data reflects documentation of their consent.

\section{Participants and design}

Sixty-eight undergraduate students (45 women, 23 men; age $M=21.54, \mathrm{SD}=4.47$ ) reported to a lecture hall for a study described as an investigation of personality and perception. They received credit toward a course requirement in exchange for their participation. Students were randomly assigned between the free writing $(n=32)$ and controlled writing $(n=36)$ conditions.

\section{Procedure}

Participants first completed a brief demographic information form, Carver and White's (1994) BIS/BAS scales, and the brief trait self-control scale (Tangney et al., 2004). In the current study, the average total score on the BAS scale was $M=40.00(\mathrm{SD}=4.48$, $\alpha=0.76)$, the average score on the BIS scale was $M=21.65$
( $\mathrm{SD}=3.51, \alpha=0.75)$, and the average score on the trait self-control scale was $42.49(\mathrm{SD}=7.35, \alpha=0.73)^{2}$.

After the questionnaires, participants completed the same 5min writing manipulation used in Study 1. Participants in the free writing condition simply wrote a story about a recent trip they had taken, whereas participants in the controlled writing condition wrote a similar story but had to avoid using the letters A or $\mathrm{N}$.

Immediately after the writing manipulation, participants completed a variant of Kimchi and Palmer's (1982, Experiment 2) local/global attentional task. The task proceeded as follows. Participants viewed stimulus triads on a viewing screen. Each of the triads depicted one standard figure at the top and two comparison figures at the bottom of the screen. The standard figure was a global square or triangle made of local squares or triangles. One comparison figure matched the global configuration of the standard and the other comparison figure matched the local elements of the standard. Participants had to indicate which of the two comparison figures (A or B) best matched the standard by circling the corresponding letter on a response sheet. Two sample stimulus triads are displayed in Figure 2.

The geometric figures for the local/global task appeared onscreen one at a time for $5 \mathrm{~s}$ each. Participants were encouraged to respond quickly with the first impression that came to mind and were assured there were no correct or incorrect answers. We counted the number of comparison figures (out of 16 triads) chosen to match the global elements of the standard, such that higher values indicate a more global (versus local) processing bias (sample $M=10.47, \mathrm{SD}=4.43$ ). Correlations among variables in this study are displayed in Table 2.

\section{RESULTS}

The central hypothesis was that the controlled writing task would increase global bias particularly among participants higher (versus lower) in BAS. It did. We regressed global attentional bias (i.e., number of global comparison figures selected on the local/global task) on writing condition, BAS scores (centered), and the Writing $\times$ BAS interaction. Neither the main effect of BAS, $b=0.08$,

${ }^{2}$ As expected, scores on the BIS/BAS and trait self-control measures did not differ as a function of experimental condition in Study 2, $t s(66)<1.32$, ps $>0.19$.

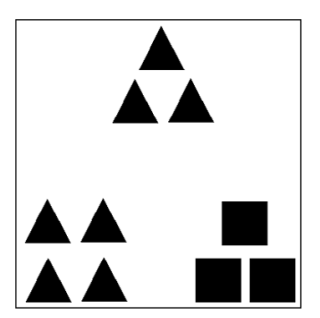

A
B

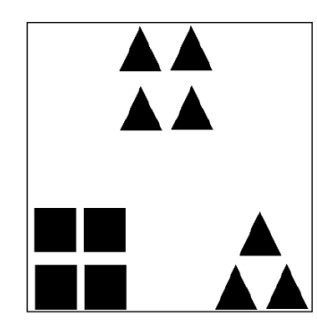

A
B
FIGURE 2 | Sample stimulus triad used in Study 2 (borrowed from Kimchi and Palmer, 1982). The standard figure at the top depicted a global square or triangle made of local squares or triangles. The comparison figures at the bottom matched either the global configuration of the standard or the local elements of the standard. Participants had to indicate which of the two comparison figures (A or B) best matched the standard. 
Table 2 | Correlations among the individual difference variables and global bias in Study 2.

\begin{tabular}{lllll}
\hline Variable & $\mathbf{1}$ & $\mathbf{2}$ & $\mathbf{3}$ & $\mathbf{4}$ \\
\hline 1. BAS & - & 0.03 & -0.20 & 0.09 \\
2. BIS & & - & -0.01 & 0.48 \\
3. Trait self-control & & & - & $-0.37^{* *}$ \\
4. Global bias & & & & -
\end{tabular}

Global bias $=$ number of global comparison figures selected on the local/global task.

$* * 0<0.001$

$t(66)=0.66, p=0.51$, nor the main effect of writing condition, $b=1.93, t(66)=1.81, p=0.07$, was statistically significant. More importantly, the predicted Writing $\times$ BAS interaction was statistically significant and in line with predictions, $b=0.49, t(66)=2.11$, $p=0.04$.

Simple slopes analyses indicated that among participants higher in BAS, the controlled writing task caused more global attention bias relative to the free writing task, $b=4.13$, $t(66)=2.77, p=0.007$. Among participants lower in BAS, the writing manipulation did not influence the selection of global comparison figures, $t<1, p=0.85$. Figure 3 displays the results based on predicted values at higher (1 SD above the mean) versus lower levels (1 SD below the mean) of BAS.

We ran the regression again, this time with trait self-control as the individual difference predictor instead of BAS. More specifically, we regressed global attentional bias on writing condition, trait self-control scores (centered), and the Writing $\times$ Trait SelfControl interaction. The main effect of writing condition was not statistically significant, $b=1.48, t(66)=1.45, p=0.15$, but the main effect of trait self-control was statistically significant, $b=-0.20, t(66)=2.93, p=0.005$. More importantly, the Writing $\times$ Trait Self-Control interaction was not significant, $b=0.18$, $t(66)=0.13, p=0.90$.

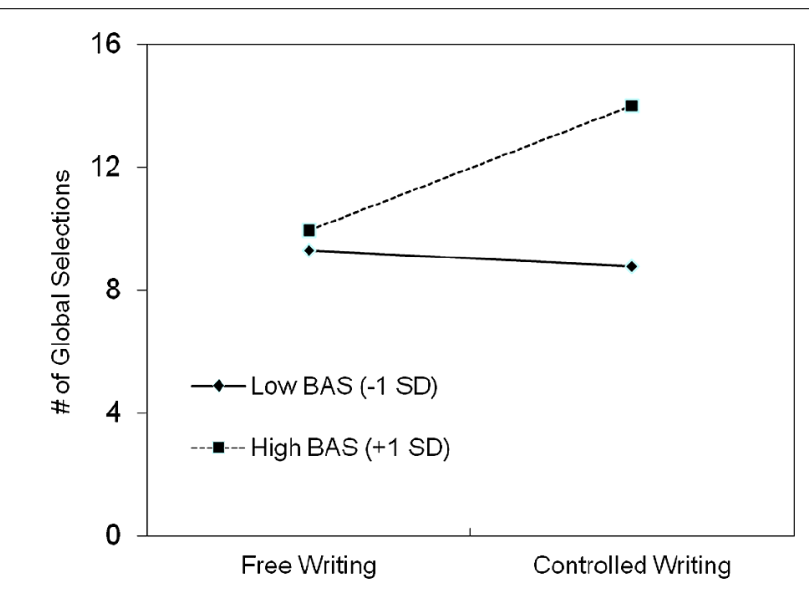

FIGURE 3 | Number of target figiues selected to match the global elements of the standard figures on Kimchi and Palmer's (1982) local/global processing task as a function of writing condition and trait approach motivation (BAS; Study 2).
With BIS as the individual difference predictor, neither the main effect of writing condition, $b=1.94, t(66)=1.83, p=0.07$, nor the main effect of BIS, $b=0.13, t(66)=0.88, p=0.38$, was statistically significant. Further, neither was the Writing $\times$ BIS interaction, $b=-0.06, t(66)=0.21, p=0.84$.

\section{DISCUSSION}

The results from Study 2 revealed that exercising self-control influences the breadth of attention for some individuals. Specifically, individuals higher in trait approach motivation showed a stronger preference for global stimulus elements after exercising (versus not exercising) self-control on a writing task. Among individuals lower in approach motivation, the exercise of self-control had no such effect. These results support the hypothesis that acts of self-control influence attention in a manner that suggests increased approach motivation.

The aftereffects of exercising self-control on global attentional bias were not moderated by trait self-control or by BIS, which suggests that self-control and behavioral inhibition did not underlie the observed effects. Unexpectedly, the zero-order correlation between trait self-control and global attentional bias was statistically significant, such that persons higher in trait selfcontrol exhibited a less pronounced global bias. Nonetheless, trait self-control did not moderate the effect of the writing manipulation whereas trait BAS did, suggesting that approach motivation but not self-control was crucial for the observed aftereffect of exercising self-control.

\section{GENERAL DISCUSSION}

Two studies examined the relationship between trait approach motivation and the aftereffects of exercising self-control. There were two major conclusions. First, exercising self-control appears to influence responses that seemingly require little or no selfcontrol. Specifically, exercising self-control caused a subsequent increase in two different responses that previously have been associated with approach motivation, namely optimism (Study 1) and attentional breadth (Study 2). Second, these aftereffects emerged particularly among individuals higher (versus lower) in trait approach motivation. These conclusions bear on several issues in the literature on self-control and ego depletion.

\section{THE AFTEREFFECTS OF SELF-CONTROL}

Most prior research on the strength model of self-control has used self-control tasks as the dependent measure. Even research that has specifically investigated the aftereffects of self-control has used dependent variables that require self-control (Friese et al., 2013). As a result, relatively little is known about the aftereffects of exercising self-control for other, non-controlled responses. Some experiments have assessed performance on tasks that did versus did not require controlled cognitive processing. For example, Schmeichel (2007) found that exercising self-control temporarily reduces working memory span but leaves simple short-term memory span intact. These and related findings (e.g., Schmeichel et al., 2003) have been interpreted as evidence that the aftereffects of self-control are specific to responses that require controlled processing. 
The current research challenges that conclusion by finding that exercising self-control influences subsequent responding that requires little or no self-control. Neither of the dependent measures used in the current studies is thought to require overriding or altering a predominant response tendency, and to the best of our knowledge neither of the dependent measures has been associated with temporary fluctuations in self-control. Rather, previous research has associated responding on these measures with state-level variations in approach motivation.

We used optimism as a dependent measure in Study 1 because previous research suggested an association between approach motivation and increased optimism. For example, approach motivation has repeatedly been associated with relatively greater activation of the left versus right hemisphere of the brain (Harmon-Jones, 2003), and Drake (1987) found more optimistic judgments for events presented to the left versus right hemisphere. Similarly, Drake and Ulrich (1992) found that a paper-and-pencil measure of left hemisphere predominance predicted greater personal optimism. The approach-related feelings of anger (Lerner et al., 2003) and power (e.g., Anderson and Galinsky, 2006) have also been associated with increased optimism. Additionally, research on individual differences in self-control and optimism suggested that the two traits are complementary insofar as they both contribute to well-being, but they operate through separate mechanisms (Carver, 2014). Given that optimism and self-control were positively correlated in Study 1 , the pattern of results we observed is the opposite of what one would expect under a typical ego depletion interpretation (i.e., after exercising self-control, self-control should be depleted and thus optimism should similarly decline). Evidence in Study 1 that exercising self-control caused an increase in optimism among individuals higher in trait approach motivation is thus more consistent with the idea that exercising self-control can increase approach-related responding.

We used attentional breadth as the dependent measure in Study 2 because research by Förster et al. (2006) had observed that a manipulation to increase approach motivation increases attentional breadth. In one representative experiment they manipulated approach versus avoidance motivation by asking participants to help a hypothetical cartoon mouse navigate a maze. In the approach motivation condition, participants imagined helping the cartoon mouse navigate the maze to obtain cheese. In the avoidance motivation condition, participants imagined helping the cartoon mouse navigate a maze to escape a lurking owl. Subsequent performance on a local/global visual processing task revealed that participants who had imagined an approachmotivated mouse responded faster to global stimuli than to local stimuli, relative to participants who had imagined an avoidancemotivated mouse. Likewise, in the current study, the prior exercise of self-control caused an increase in global attentional bias among individuals higher in trait approach motivation. We take this as evidence that exercising self-control can increase approach-motivated responding.

Approach motivation has also been found to reduce attentional breadth. Specifically, Gable and Harmon-Jones (2008) proposed that biologically based approach-motivated states, such as those associated with sexual behavior or the consumption of food and water, may reduce attentional breadth as organisms focus on obtaining a desired object. Based on this reasoning, Gable and Harmon-Jones conducted a series of experiments that found evidence of reduced attentional breadth under high approach motivation. In one representative experiment they manipulated approach motivation by having participants view pictures. In the high approach condition participants viewed a series of appetitive object pictures (i.e., desserts) whereas in the low approach group they viewed neutral object pictures (i.e., rocks). All participants then completed a local/global visual processing task as a measure of attentional breadth. The results indicated that viewing appetitive dessert pictures (compared to neutral rock pictures) reduces the preference for global stimulus features on a local/global task. These findings were subsequently replicated in research examining neural activations associated with approach motivation (Harmon-Jones and Gable, 2009).

If approach motivation can increase or decrease attentional breadth, then why did we find that exercising self-control increases attentional breadth in Study 2? We suspect that in the absence of salient appetitive stimuli (as was the case in Study 2), exercising self-control induces a low-intensity approach motivational state akin to the one that arises when imagining a mouse chase cheese (Förster et al., 2006), as opposed to the more high-intensity approach state that occurs when hungry persons view pictures of delectable desserts (Gable and Harmon-Jones, 2008). If our suspicions are correct, and the salience of appetitive stimuli is crucial for understanding when approach motivation will broaden attention, then future research may find that exercising self-control can reduce attentional breadth when appetitive or rewarding stimuli are salient in the immediate environment.

Another possibility was suggested by the findings of Gable and Harmon-Jones (2011). They assessed attentional breadth before and after attaining a goal, and they found that pre-goal (high approach) positive affect was related to local focus, whereas postgoal (low approach) positive affect was related to global focus. If completing the controlled writing task in Study 2 can be construed as a goal, then a low approach-motivated post-goal state and its attendant global focus would be expected to emerge following task completion. This is precisely what we observed among participants higher in BAS.

Insofar as the current research was specifically interested in the approach-related aftereffects of exercising self-control, it would be reasonable to expect main effects of trait approach motivation on the dependent measures in the current studies. However, neither study found a significant relationship between trait BAS and the dependent variables-BAS only predicted optimism and attentional breadth after participants had exercised self-control. This pattern suggests that trait BAS may be especially predictive of approach-related responding under "activating" or "hot" conditions. The current findings are consistent with evidence that experimental inductions of approach-motivated states can bias attention and increase optimism (Lerner et al., 2003; Förster et al., 2006). We are aware of no prior evidence that high levels of trait approach motivation have the same effects. The implication is that exercising self-control creates a context that is conducive to approach-related responding, especially among those higher 
in trait approach motivation. We propose that exercising selfcontrol on an innocuous writing task may induce a low-level intensity of approach motivation that, combined with higher levels of trait approach motivation, contributes to the increases in optimism and breadth of attention observed in the present studies.

Moreover, in neither study did trait self-control moderate the aftereffects of exercising self-control. These null results suggest that self-control was not the main process by which performing the controlled writing task influenced optimism and global attentional bias. The logic underlying this conclusion is as follows. To the extent that a particular process underlies an experimental effect, individual differences in the tendency to engage the process should moderate the effect. Hence, if self-control were the key to understanding the aftereffects of exercising self-control in the current studies, then individual differences in trait self-control would have moderated the effects of the writing manipulation because trait self-control is directly related to the tendency to engage self-control (e.g., Schmeichel and Zell, 2007). However, the results from both studies indicated that the increases in optimism and attentional breadth after exercising self-control were moderated by trait approach motivation, not by trait self-control. We acknowledge that for a different class of dependent measures (e.g., dependent measures that require the person to override a response), individual differences in self-control are likely to be a moderating variable, which would suggest that self-control is key to observed changes in such measures (e.g., Gröpel et al., 2014).

Taken together, the current results indicate that exercising selfcontrol can influence non-controlled responding in addition to its well-known consequences for further efforts at self-control. We propose this is because exercising self-control not only reduces self-control strength but can also influence the other side of the self-control struggle-impulse strength.

ROLE OF IMPULSE STRENGTH IN THE AFTEREFFECTS OF SELF-CONTROL Revised reinforcement sensitivity theory (RST) may help to explain why exercising self-control could both reduce self-control strength and increase approach-motivated impulse strength. According to revised RST (Gray and McNaughton, 2000; Corr, 2008), three coordinated systems regulate behavior. One is the BAS, which is strongly related to approach motivation and mediates responding to appetitive stimuli; second is the fightflight-freeze system (FFFS), which underlies reactions to aversive stimuli; third is the BIS, which detects and helps to resolve conflicts that arise within and between the other two systems. According to the "joint subsystems hypothesis" (Corr, 2008), the BIS and the BAS have a reciprocal relationship. Research has found that exercising self-control temporarily reduces BIS-related activation (Inzlicht and Gutsell, 2007). Based on revised RST, then, one would expect that exercising self-control can also amplify BAS, because, to put it simply, as the BIS is weakened, the BAS becomes relatively unconstrained. The current findings are consistent with this view insofar as exercising self-control in a way that has been found to reduce self-control strength in previous research (e.g., Mead et al., 2009; Schmeichel and Vohs, 2009) caused an increase in approach-related responding in the current studies, especially for participants prone to exhibit approach-motivated impulses.

Another possible explanation for why exercising self-control increases approach-motivated responding centers on the emotional consequences of exercising self-control. As we noted at the outset of this article, exerting self-control can be a chore, and a recent meta-analysis found evidence that exercising self-control increases negative affect, although the effect is small and inconsistent across studies (Hagger et al., 2010). We suggest that after exertion (perhaps particularly an unrewarded exertion) on a selfcontrol task, motivational orientation shifts in the direction of increased reward-seeking behavior (see Inzlicht and Schmeichel, 2012). This shift in motivational orientation may be associated with an increase in frustration associated with exercising selfcontrol (Gal and Liu, 2011). If that is correct, then increased frustration and anger could help to explain the current findings as both emotions can be considered approach-motivated emotions (e.g., Harmon-Jones, 2003) and have been linked to increased attention to reward (Ford et al., 2010). It is a limitation of the current research that participants' subjective states were not measured in either study. Future research should consider the extent to which anger and frustration in response to exercising self-control helps to explain the cognitive and behavioral aftereffects.

One potential alternative explanation for the current findings is that prior acts of self-control reveal personality traits or motivational impulses generally, rather than approach-motivated impulses specifically (Baumeister etal., 2006). The research reported here was designed specifically to examine approach motivation and approach-motivated responding. We did not assess responses associated with other traits, so additional research is needed before the more general claim can be assessed. Based on revised RST (Gray and McNaughton, 2000; Corr, 2008) one could derive the prediction that a reduction in BIS-related activation loosens the reins on the FFFS and thereby potentiates responses to threatening stimuli, too, and presumably this would be most true for individuals prone to experience fear and anxiety.

Although the current studies focused on the aftereffects of self-control for approach-motivated responses, the results may have implications for the well-known aftereffects for further selfcontrol. Conflict is inherent to self-control (e.g., Hofmann et al., 2012a), and this conflict can be experienced as unpleasant or aversive. To succeed at self-control may require success at tolerating or otherwise coping with this aversive state. If exercising self-control increases approach motivation, then one may be prone to commit to responses that hasten reward or good feelings and thereby alleviate the aversive state. In this view, exercising self-control causes a shift in motivational orientation (i.e., increased approach) that may help to resolve inner conflict and minimize aversive emotions, but at the same time increase the likelihood of failing at self-control.

\section{LIMITATIONS AND FUTURE DIRECTIONS}

One limitation of the current work is that participants in each study completed the same initial exercise of self-control (i.e., the controlled writing task). One impressive element of previous 
research on the strength model of self-control is that many different self-control exercises, from controlled writing to thought suppression to emotion exaggeration, have been observed to have similar aftereffects. We assume the same is true for the effects of exercising self-control on approach-motivated responding, but additional research using different initial exercises of self-control is needed to verify this assumption. One previous study found that suppressing emotional expressions led to an increase in selfreported approach motivation (Schmeichel et al., 2010), which suggests that the approach-motivated aftereffects of exercising selfcontrol are not specific to the controlled writing task used in the current studies.

Rather than varying the types of self-control participants exercised on the initial tasks, in the current studies we varied the dependent measures across studies. The dependent measures we chose were not direct measures of approach motivation but instead are measures that have been found in previous research to relate to approach motivational states. Future research should strive to measure approach motivation more directly. Research along these lines has already begun to emerge. For example, a study by Wagner etal. (2013) found that prior acts of selfcontrol cause both reduced activity in brain centers associated with self-control (i.e., inferior frontal gyrus) and increased activity in brain centers associated with reward (i.e., orbitofrontal cortex).

Furthermore, it must be true that the dependent measures we used are influenced by processes other than approach motivation. Nonetheless, based on prior evidence suggesting that the dependent measures we used are influenced by approach-motivated states, and given the consistency of results across the two measures, we believe the most parsimonious conclusion is that exercising selfcontrol increases approach-motivated responding, particularly for individuals higher in trait approach motivation. Individuals lower in trait approach motivation appeared relatively impervious to the aftereffects of self-control, but the extent to which their other (i.e., non-approach-related) responses are affected by exercising self-control remains to be established.

\section{CONCLUSION}

The current investigation found that the aftereffects of self-control extend beyond further acts of control. The common thread for the aftereffects observed here was approach motivation, a relationship corroborated by evidence of stronger effects among persons higher in trait approach motivation. We conclude, in accordance with revised RST, that exercising self-control may both reduce self-control strength and trigger an increase in approachmotivated impulse strength. This increase in approach motivation suggests the potential for widespread aftereffects of overriding a response that cannot be captured by measures of self-control alone.

\section{ACKNOWLEDGMENTS}

Adrienne Crowell, Nicholas J. Kelley, and Brandon J. Schmeichel, Department of Psychology, Texas A\&M University. This work was supported in part by the National Science Foundation [BCS0921276]. Thank you to Miki Alvarado, Rachel Gandy, Nichole Montalbano, Rebekah Nichols, Raymond Serra, and Alyssa Yates for helping to conduct these studies and to David Tang for providing helpful feedback on this work. The open access publishing fees for this article have been covered by the Texas A\&M University Online Access to Knowledge (OAK) Fund, supported by the University Libraries and the Office of the Vice President for Research.

\section{REFERENCES}

Anderson, C., and Galinsky, A. D. (2006). Power, optimism, and risk-taking. Eur. J. Soc. Psychol. 36, 511-536. doi: 10.1002/ejsp.324

Baumeister, R. F., Bratslavsky, E., Muraven, M., and Tice, D. M. (1998). Ego depletion: is the active self a limited resource? J. Pers. Soc. Psychol. 74, 1252-1265. doi: 10.1037/0022-3514.74.5.1252

Baumeister, R. F., Gailliot, M., DeWall, C. N., and Oaten, M. (2006). Self-regulation and personality: how interventions increase regulatory success, and how depletion moderates the effects of traits on behavior. J. Pers. 74, 1773-1801. doi: 10.1111/j.1467-6494.2006.00428.x

Beaver, J. D., Lawrence, A. D., van Ditzhuijzen, J., Davis, M. H., Woods, A., and Calder, A. J. (2006). Individual differences in reward drive predict neural responses to images of food. J. Neurosci. 26, 5160-5166. doi: 10.1523/JNEUROSCI.035006.2006

Carver, C. S. (2005). Impulse and constraint: perspectives from personality psychology, convergence with theory in other areas, and potential for integration. Pers. Soc. Psychol. Rev. 9, 312-333. doi: 10.1207/s15327957pspr0904_2

Carver, C. S. (2014). Self-control and optimism are distinct and complementary strengths. Pers. Individ. Dif. 66, 24-26. doi: 10.1016/j.paid.2014.02.041

Carver, C. S., and Harmon-Jones, E. (2009). Anger is an approach-related affect: evidence and implications. Psychol. Bull. 135, 183-204. doi: 10.1037/a0013965

Carver, C. S., and White, T. L. (1994). Behavioral inhibition, behavioral activation, and affective responses to impending reward and punishment: the BIS/BAS scales. J. Pers. Soc. Psychol. 67, 319-333. doi: 10.1037/0022-3514.67.2.319

Christiansen, P., Cole, J. C., and Field, M. (2012). Ego depletion increases ad-lib alcohol consumption: investigating cognitive mediators and moderators. Exp. Clin. Psychopharmacol. 20, 118-128. doi: 10.1037/a0026623

Corr, P. (2008). The Reinforcement Sensitivity Theory of Personality. Cambridge: Cambridge University Press.

Denson, T. F., Pedersen, W. C., Friese, M., Hahm, A., and Roberts, L. (2011). Understanding impulsive aggression: angry rumination and reduced self-control capacity are mechanisms underlying the provocation-aggression relationship. Pers. Soc. Psychol. Bull. 37, 850-862. doi: 10.1177/0146167211401420

DeWall, C. N., Baumeister, R. F., Stillman, T. F., and Gailliot, M. T. (2007). Violence restrained: effects of self-regulation and its depletion on aggression. J. Exp. Soc. Psychol. 43, 62-76. doi: 10.1016/j.jesp.2005.12.005

Drake, R. A. (1987). Conceptions of own versus others' outcomes: manipulation by monaural attentional orientation. Eur. J. Soc. Psychol. 17, 373-375. doi: 10.1002/ejsp. 2420170313

Drake, R. A., and Ulrich, G. (1992). Line bisecting as a predictor of personal optimism and desirability of risky behaviors. Acta Psychol. 79, 219-226. doi: 10.1016/0001-6918(92)90058-L

Finkel, E. J., DeWall, C. N., Slotter, E. B., Oaten, M., and Foshee, V. A. (2009). Self-regulatory failure and intimate partner violence perpetration. J. Pers. Soc. Psychol. 97, 483-499. doi: 10.1037/a0015433

Fischer, P., Greitemeyer, T., and Frey, D. (2008). Self-regulation and selective exposure: the impact of depleted self-regulation resources on confirmatory information processing. J. Pers. Soc. Psychol. 94, 382-395. doi: 10.1037/00223514.94.3.382

Ford, B. Q., Tamir, M., Brunye, T. T., Shirer, W. R., Mahoney, C. R., and Taylor, H. A. (2010). Keeping your eyes on the prize: anger and visual attention to threats and rewards. Psychol. Sci. 21, 1098-1105. doi: 10.1177/0956797610375450

Förster, J., Friedman, R., Özelsel, A., and Denzler, M. (2006). Enactment of approach and avoidance behavior influences the scope of perceptual and conceptual attention. J. Exp. Soc. Psychol. 42, 133-146. doi: 10.1016/j.jesp.2005. 02.004

Freeman, N., and Muraven, M. (2010). Self-control depletion leads to increased risk taking. Soc. Psychol. Pers. Sci. 2, 175-181.

Friese, M., Binder, J., Luechinger, R., Boesiger, P., and Rasch, B. (2013). Suppressing emotions impairs subsequent stroop performance and reduces 
prefrontal brain activation. PLOS ONE 8:e60385. doi: 10.1371/journal.pone. 0060385

Gable, P. A., and Harmon-Jones, E. (2008). Approach-motivated positive affect reduces breadth of attention. Psychol. Sci. 19, 476-482. doi: 10.1111/j.14679280.2008.02112.x

Gable, P. A., and Harmon-Jones, E. (2011). Attentional consequences of pregoal and postgoal positive affects. Emotion 11, 1358-1367. doi: 10.1037/a0025611

Gal, D., and Liu, W. (2011). Grapes of wrath: the angry effects of self-control. J. Consum. Psychol. 38, 445-458.

Gasper, K., and Clore, G. (2002). Attending to the big picture: mood and global versus local processing of visual information. Psychol. Sci. 13, 34-40. doi: 10.1111/1467-9280.00406

Gohm, C. L., and Clore, G. L. (2000). Individual differences in emotional experience: mapping available scales to processes. Pers. Soc. Psychol. Bull. 26, 679-697. doi: $10.1177 / 0146167200268004$

Gray, J., and McNaughton, N. (2000). The Neuropsychology of Anxiety, 2nd Edn. New York, NY: Oxford University Press.

Gröpel, P., Baumeister, R. F., and Beckmann, J. (2014). Action versus state orientation and self-control performance after depletion. Pers. Soc. Psychol. Bull. 40, 476-487. doi: 10.1177/0146167213516636

Hagger, M. S., Wood, C., Stiff, C., and Chatzisarantis, N. L. D. (2010). Ego depletion and the strength model of self-control: a meta-analysis. Psychol. Bull. 136, 495525. doi: 10.1037/a0019486

Harmon-Jones, E. (2003). Clarifying the emotive functions of asymmetrical frontal cortical activity. Psychophysiology 40, 838-848. doi: 10.1111/1469-8986.00121

Harmon-Jones, E., and Gable, P. A. (2009). Neural activity underlying the effect of approach-motivated positive affect on narrowed attention. Psychol. Sci. 20, 406-409. doi: 10.1111/j.1467-9280.2009.02302.x

Harmon-Jones, E., Harmon-Jones, C., and Price, T. F. (2013). What is approach motivation? Emot. Rev. 5, 291-295. doi: 10.1177/175407391 3477509

Hofmann, W., Baumeister, R. F., Förster, G., and Vohs, K. D. (2012a). Everyday temptations: an experience sampling study of desire, conflict, and self-control. J. Pers. Soc. Psychol. 102, 1318-1335. doi: 10.1037/a0026545

Hofmann, W., Vohs, K. D., and Baumeister, R. F. (2012b). What people desire, feel conflicted about, and try to resist in everyday life. Psychol. Sci. 23, 582-588. doi: 10.1177/0956797612437426

Hofmann, W., Friese, M., and Strack, F. (2009). Impulse and self-control from a dual-systems perspective. Perspect. Psychol. Sci. 4, 162-176. doi: 10.1111/j.17456924.2009.01116.x

Inzlicht, M., and Gutsell, J. (2007). Running on empty: neural signals for self-control failure. Psychol. Sci. 18, 933-937. doi: 10.1111/j.1467-9280.2007.02004.x

Inzlicht, M., and Schmeichel, B. J. (2012). What is ego depletion? Toward a mechanistic revision of the resource model of self-control. Perspect. Psychol. Sci. 7, 450-463. doi: 10.1177/1745691612454134

Inzlicht, M., Schmeichel, B. J., and Macrae, C. N. (2014). Why self-control seems (but may not be) limited. Trends Cogn. Sci. 18, 127-133. doi: 10.1016/j.tics.2013. 12.009

Kimchi, R., and Palmer, S. E. (1982). Form and texture in hierarchically constructed patterns. J. Exp. Psychol. Hum. Percept. Perform. 8, 521-535. doi: 10.1037/00961523.8.4.521

Lerner, J. S., Gonzalez, R. M., Small, D. A., and Fischoff, B. (2003). Effects of fear and anger on perceived risks of terrorism: a national field experiment. Psychol. Sci. 14, 144-150. doi: 10.1111/1467-9280.01433

Lewandowski, G. W., Ciarocco, N. J., and Pettanato, M. (2012). Pick me up: ego depletion and receptivity to relationship initiation. J. Soc. Pers. Relat. 29, 10711084. doi: 10.1177/0265407512449401

Mead, N. L., Baumeister, R. F., Gino, F., Schweitzer, M. E., and Ariely, D. (2009). Too tired to tell the truth: self-control resource depletion and dishonesty. J. Exp. Soc. Psychol. 45, 594-597. doi: 10.1016/j.jesp.2009.02.004

Muraven, M., and Baumeister, R. F. (2000).Self-regulation and depletion of limited resources: does self-control resemble a muscle? Psychol. Bull. 126, 247-259. doi: 10.1037/0033-2909.126.2.247
Muraven, M., Collins, R. L., and Nienhaus, K. (2002). Self-control and alcohol restraint: an initial application of the self-control strength model. Psychol. Addict. Behav. 16, 113-120. doi: 10.1037/0893-164X. 16.2.113

Perloff, L. S., and Fetzer, B. K. (1986). Self-other judgments and perceived vulnerability to victimization. J. Pers. Soc. Psychol. 50, 502-510. doi: 10.1037/00223514.50.3.502

Salmon, S. J., Adriaanse, M. A., De Vet, E., Fennis, B. M., and De Ridder, D. T. D. (2014). When the going gets tough who keeps going? Depletion sensitivity moderates the ego-depletion effect. Front. Psychol. 5:647. doi: 10.3389/fpsyg.2014.00647

Schmeichel, B. J. (2007). Attention control, memory updating, and emotion regulation temporarily reduce the capacity for executive control. J. Exp. Psychol. Gen. 136, 241-255. doi: 10.1037/0096-3445.136.2.241

Schmeichel, B. J., Harmon-Jones, C., and Harmon-Jones, E. (2010). Exercising selfcontrol increases approach motivation. J. Pers. Soc. Psychol. 99, 162-173. doi: 10.1037/a0019797

Schmeichel, B. J., and Vohs, K. (2009). Self-affirmation and self-control: affirming core values counteracts ego depletion. J. Pers. Soc. Psychol. 96, 770-782. doi: 10.1037/a0014635

Schmeichel, B. J., Vohs, K. D., and Baumeister, R. F. (2003). Intellectual performance and ego depletion: role of the self in logical reasoning and other information processing. J. Pers. Soc. Psychol. 85, 33-46. doi: 10.1037/0022-3514.85.1.33

Schmeichel, B. J., and Zell, A. (2007). Trait self-control predicts performance on behavioral tests of self-control. J. Pers. 75, 743-756. doi: 10.1111/j.14676494.2007.00455.x

Sedikides, C., and Green, J. D. (2000). On the self-protective nature of inconsistency/negativity management: using the person memory paradigm to examine self-referent memory. J. Pers. Soc. Psychol. 79, 906-922. doi: 10.1037/00223514.79.6.906

Stucke, T. S., and Baumeister, R. F. (2006). Ego depletion and aggressive behavior: is the inhibition of aggression a limited resource? Eur. J. Soc. Psychol. 36, 1-13. doi: 10.1002/ejsp.285

Tangney, J. P., Baumeister, R. F., and Boone, A. L. (2004). High self-control predicts good adjustment, less pathology, better grades, and interpersonal success. J. Pers. 72, 271-324. doi: 10.1111/j.0022-3506.2004.00263.x

Underwood, B. J. (1975). Individual differences as a crucible in theory construction. Am. Psychol. 30, 128-134. doi: 10.1037/h0076759

Vohs, K. D., and Heatherton, T. F. (2000). Self-regulatory failure: a resourcedepletion approach. Psychol. Sci. 11, 249-254. doi: 10.1111/1467-9280. 00250

Wagner, D. D., Altman, M., Boswell, R. G., Kelley, W. M., and Heatherton, T. F. (2013). Self-regulatory depletion enhances neural responses to rewards and impairs top-down control. Psychol. Sci. 11, 2262-2271. doi: $10.1177 / 0956797613492985$

Conflict of Interest Statement: The authors declare that the research was conducted in the absence of any commercial or financial relationships that could be construed as a potential conflict of interest.

Received: 08 August 2014; accepted: 12 September 2014; published online: 29 September 2014.

Citation: Crowell A, Kelley NJ and Schmeichel BJ (2014) Trait approach motivation moderates the aftereffects of self-control. Front. Psychol. 5:1112. doi: 10.3389/fpsyg.2014.01112

This article was submitted to Personality and Social Psychology, a section of the journal Frontiers in Psychology.

Copyright (C) 2014 Crowell, Kelley and Schmeichel. This is an open-access article distributed under the terms of the Creative Commons Attribution License (CC BY). The use, distribution or reproduction in other forums is permitted, provided the original author(s) or licensor are credited and that the original publication in this journal is cited, in accordance with accepted academic practice. No use, distribution or reproduction is permitted which does not comply with these terms. 